\title{
Micro-PIV Measurements of Multiphase Flow in Deforming Porous Media Subject to Mineral Dissolution
}

\author{
R. Molla ${ }^{1}$, N. Raventhiran ${ }^{1}$, Y. $\mathbf{L i}^{1} *$ \\ ${ }^{1}$ Montana State University, Mechanical\&Industrial Engineering, Bozeman, USA \\ *yaofa.li@montana.edu
}

\begin{abstract}
Mineral dissolution is studied in novel calcite-based porous micromodels under single- and multiphase conditions, with a focus on the interactions of mineral dissolution with pore flow. Microscopic particle image velocimetry (PIV) was utilized to simultaneously characterize the local velocity field and the instantaneous shapes of the dissolving grains. The preliminary results provide a unique view of the coupled dynamics between pore flow and mineral dissolution.
\end{abstract}

\section{Introduction}

Mineral dissolution in porous media coupled with single- or multi-phase flows is pervasive in natural and engineering systems. In subsurface environments, the solid porous matrix is composed of various types of minerals, through which subsurface water flows. Dissolution of minerals occurs as chemicals in the solid phase is transformed into ions in the aqueous phase, effectively modifying the physical, hydrological and geochemical properties of the solid matrix as well as the chemistry in the aqueous phase Daccord (1987). These processes play a defining role in a broad range of applications including carbon capture and sequestration (CCS) Ott and Oedai (2015) and acid stimulation in reservoir engineering Daccord (1987). For instance, CCS is considered as a viable technology to reduce carbon emissions to the atmosphere, thus effectively mitigating global climate change. However, injection of $\mathrm{CO}_{2}$ into geologic formations leads to dissolution of minerals comprising reservoirs rocks, potentially creating leakage pathways that threaten the safety and security of $\mathrm{CO}_{2}$ storage Ott and Oedai (2015). Therefore, to successfully model, predict, control and optimize these many processes, a comprehensive understanding of mineral dissolution is crucial.

Mineral dissolution in porous media is subject to chemical reaction and transport via advection and diffusion. On the one hand, pore flow plays a defining role in mixing and transporting the reactants to the reaction sites as well as transporting reaction products away, significantly affecting the mineral dissolution rate. On the other hand, mineral dissolution continuously modifies the porous media both structurally and chemically, which in turn reshapes the pore flow. Therefore, local dissolution rate and pore-scale flow are strongly coupled. However, our fundamental understanding of this coupling effect at the pore level is still limited, leading to strong challenges in the effort of predicting mineral dissolution at much larger scales.

\section{Experimental Methods}

The micromodels used in the experiments were fabricated in calcite as shown in Figure 1/Li et al. (2017). To fabricate a calcite-based micromodel, the porous patterns, which is inspired by real geological structures, was precisely formed in calcite substrate using photolithography and wet etching. The etched substrate was then bonded with adhesive to a glass slide to form a micromodel, to which nanoports were attached, to serve as fluid delivery ports. These surrogate porous media offer precise control over the structures and chemical properties, and facilitate unobstructed and unaberrated optical access to the pore flow with microPIV methods. White field microscopy and the fluorescent micro-PIV method were simultaneously employed by seeding the water phase with fluorescent particles, in order to achieve simultaneous measurement of the velocity fields of the aqueous and structure evolution of the solid phase 


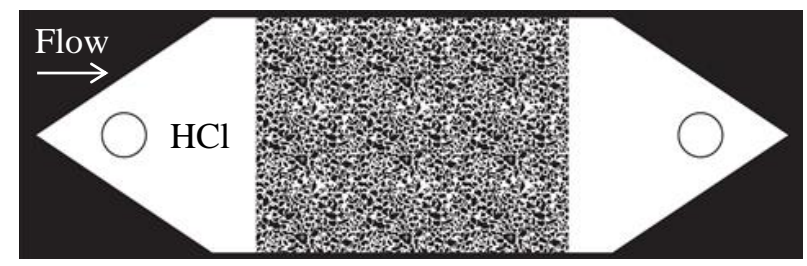

Figure 1: The calcite-based micromodel consiting of an inlet, a porous section, and an outlet.

\section{Results}

The preliminary results provide a unique view of the flow dynamics during mineral dissolution. Figures $2 \mathrm{a}$ and $2 \mathrm{p}$ show the sample velocity fields of the pore-scale flow under single and multiphase flow conditions, respectively. When $\mathrm{HCl}$ concentration is low but the pore flow rate is relatively high, the reaction product (i.e., $\mathrm{CO}_{2}$ ) is instantaneously dissolved in the aqueous phase leading to a single phase flow. However, when $\mathrm{HCl}$ concentration is high such that the produced $\mathrm{CO}_{2}$ cannot be instantaneously dissolved, it will emerge as a separate phase, leading to a multiphase flow. While the flow field with single-phase flow is relatively simple, that flow is significantly modified in the multiphase flow case due to the presence $\mathrm{CO}_{2}$ bubbles that are generated in-situ as a result of chemical reaction between the liquid and solid phases. The separate $\mathrm{CO}_{2}$ phase is expected to not only divert the $\mathrm{HCl}$ flow, but also shied the solid surfaces from further reaction, thus significantly modifying the local dissolution pattern and rate.

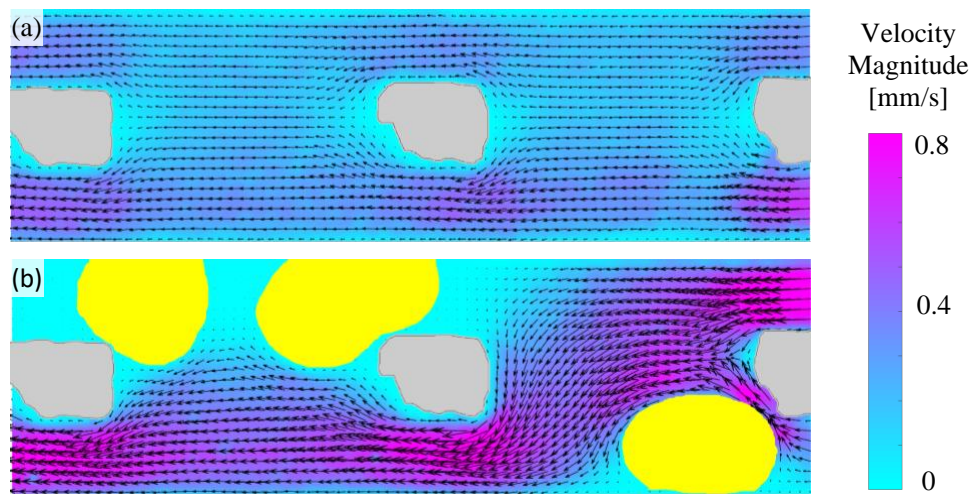

Figure 2: Sample flow fields under single-phase (a) and multiphase (b) flow conditions, showing water velocities as color contours; gas and calcite grains as yellow and grey regions, respectively.

\section{Acknowledgements}

This work was performed in part at the Montana Nanotechnology Facility, an NNCI facility supported by NSF Grant ECCS-1542210, and with support by the Murdock Charitable Trust. YL thanks the Norm Asbjornson College of Engineering and the Center for Faculty Excellence at Montana State University.

\section{References}

Daccord G (1987) Chemical dissolution of a porous medium by a reactive fluid. Physical review letters $58: 479$

Li Y, Kazemifar F, Blois G, and Christensen KT (2017) Micro-piv measurements of multiphase flow of water and liquid $\mathrm{CO}_{2}$ in 2-d heterogeneous porous micromodels. Water Resources Research 53:6178-6196

Ott $\mathrm{H}$ and Oedai S (2015) Wormhole formation and compact dissolution in single-and two-phase $\mathrm{CO}_{2}$-brine injections. Geophysical Research Letters 42:2270-2276 